\title{
INSIGNIA: Beekeepers as citizen scientists investigate the environment of their honey bees
}

\author{
Robert Brodschneidera, ${ }^{*}$, Kristina Gratzera, Norman L Carreck ${ }^{b c}$, Flemming \\ Vejsnaes ${ }^{d}$, Jozef van der Steen ${ }^{e}$ \\ aInstitute of Biology, University of Graz, \\ Universitätsplatz 2, 8010 Graz, Austria \\ bCarreck Consultancy Ltd, \\ Woodside Cottage, Dragons Lane, Shipley, West Sussex, RH13 8GD, UK \\ cLaboratory of Apiculture and Social Insects, University of Sussex, \\ Falmer, Brighton, East Sussex, BN1 9QG, UK \\ dDanish Beekeepers Association, \\ Fulbyvej 15, 4180 Sorø, Denmark \\ eAlveus AB Consultancy, \\ Kerkstraat 96, 5061 EL Oisterwijk, Netherlands
}

E-mail: robert.brodschneider@uni-graz.at, kristina.gratzer@uni-graz.at, norman.carreck@btinternet.com,fv@biavl.dk,alveusab@outlook.com

Beekeepers can be valuable volunteers in large scale research studies. They own honey bee (Apis mellifera) colonies, have basic equipment, and are knowledgeable about apiculture. In the INSIGNIA project, citizen science beekeepers collected samples from their colonies over two seasons. Several protocols and different sampling devices for beekeeper participation in monitoring the environment for pesticides and bee forage sources were developed and tested, including all steps until sample analysis in the laboratory. The study was accompanied by studies investigating the motivations and skills of beekeepers volunteering as citizen scientists. The samples and information collected with the help of citizen scientists will be used to model the biodiversity of pollen sources, risk mapping for pollinators and environmental pollution in different environments. 


\section{Introduction}

INSIGNIA (cItizeN Science InvestiGatioN for PesticIdes in Apicultural Products) is a European Union funded project, initiated and commissioned by the European Commission, DG SANTE, to develop a protocol for beekeepers participating in monitoring the environment for pesticides and flowering plants. Various methods were applied by 17 citizen scientists from four countries in year one and 81 citizen scientists from nine countries in year two, including all steps until sample analysis in the laboratory. During development of the citizen science protocols, upscaling to larger number of citizen scientists was always considered.

Honey bees collect nectar and pollen from flowers, and thereby bring substances from the environment to their nest, where those can be sampled. Pollen contains genetic information on the plant species flowering around the hive but could also carry environmental contaminants. Given their foraging range of several kilometers, honey bees monitor a large area around the hive [1], [2]. Beekeepers as citizen scientists have already proven their ability to collect pollen samples for scientific studies [3]. The citizen scientists' participation in INSIGNIA can be classified as a contributory project according to the classification of Shirk et al. [4].

\section{Project overview}

\subsection{Beekeeper recruitment and instruction as citizen scientists}

Calls for beekeepers to volunteer as citizen scientists were publicised in beekeeping magazines, websites, social media, beekeeper meetings or through personal contacts. Volunteers were chosen according to factors such as availability of equipment and suitable location of their apiaries. All materials not commonly found in a beekeeping operation, like sampling devices, were sent to the beekeepers prior to the study. A pamphlet with illustrated step-by-step instructions for sampling and online instruction videos were also supplied.

Communication with citizen scientists during the season was accomplished with a multilanguage closed (personalized) survey tool. Ten samplings were scheduled from late April to early September. Citizen scientists could on each sampling period choose one day from Thursday to Sunday which was convenient to them and with hopefully suitable weather conditions. On Tuesday before sampling, an 'invitation' email was sent to all participants. This contained a link to submit accompanying information of sampling success, like colony conditions, duration and exact date of sampling, flowering sources at their vicinity, or possible chemicals applied to colonies during Varroa control. In each country, a coordinator was available via various communication channels to provide further information about the study and answer questions.

\subsection{The study seasons}

From April to September 2019 (first season), four different methods of sampling the hives were developed and tested by four or five beekeepers in each of four countries (Austria, Denmark, Greece, UK). Each beekeeper participated with three colonies in one permanent apiary location.

Two methods were identified as best suitable and applied in the second study season 2020 . Based on statistical evaluations of the lab results, the study design was further improved by 
reducing the number of colonies sampled by each citizen scientist to two. The number of participants was increased to nine in each of nine countries (figure 1).

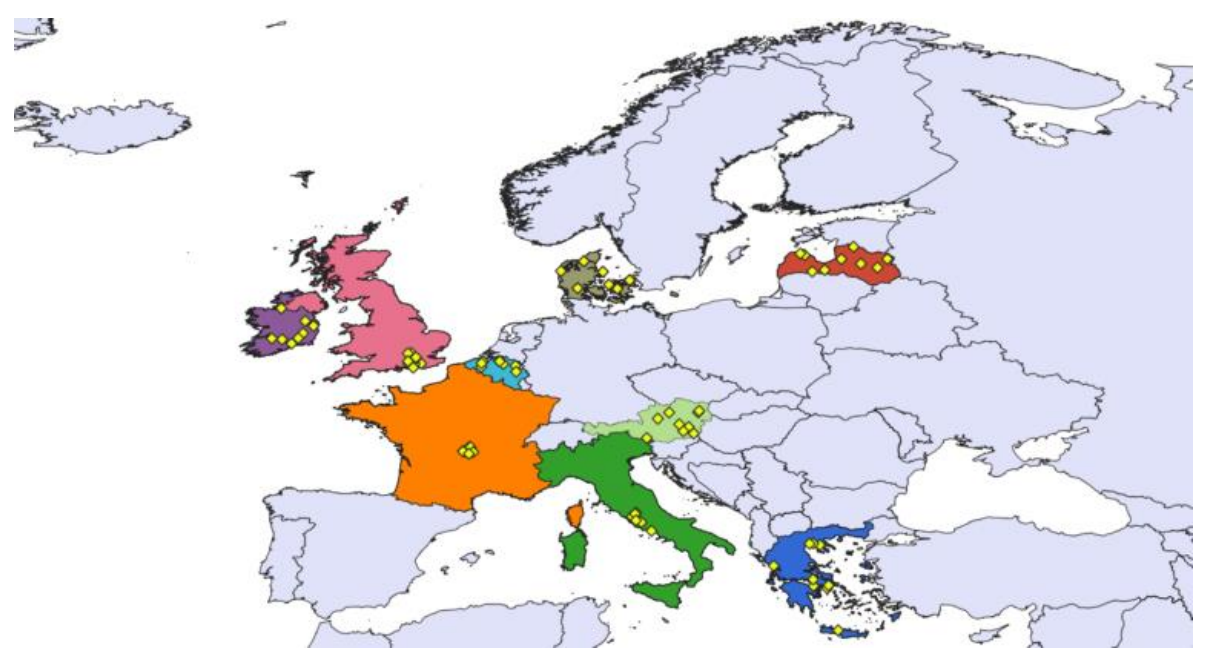

Figure 1. The 81 regular citizen scientists' apiary locations in the 2020 INSIGNIA study season.

\subsection{Different sampling methods}

In 2019, four different methods of collecting samples from the colonies were tested and compared for their suitability for citizen scientists and uptake and preservation of environmental compounds. Pollen from returning pollen forager bees can be collected with pollen traps without opening the hive. This technique is commonly used by beekeepers to produce pollen for the market (Figure 2). When bees store the collected pollen in combs, it is called beebread. Beebread is also a marketable product, but the hive must be opened to collect it with plastic straws. Two sampling techniques were newly developed or adopted for this project: Beehold tubes collect substances from bees' bodies as they pass through to enter the hive [5]. Newly developed APIStrips are impregnated with Tenax, which binds to substances circulating in the hive. These strips are inserted in a beelane of the hive [6]. 

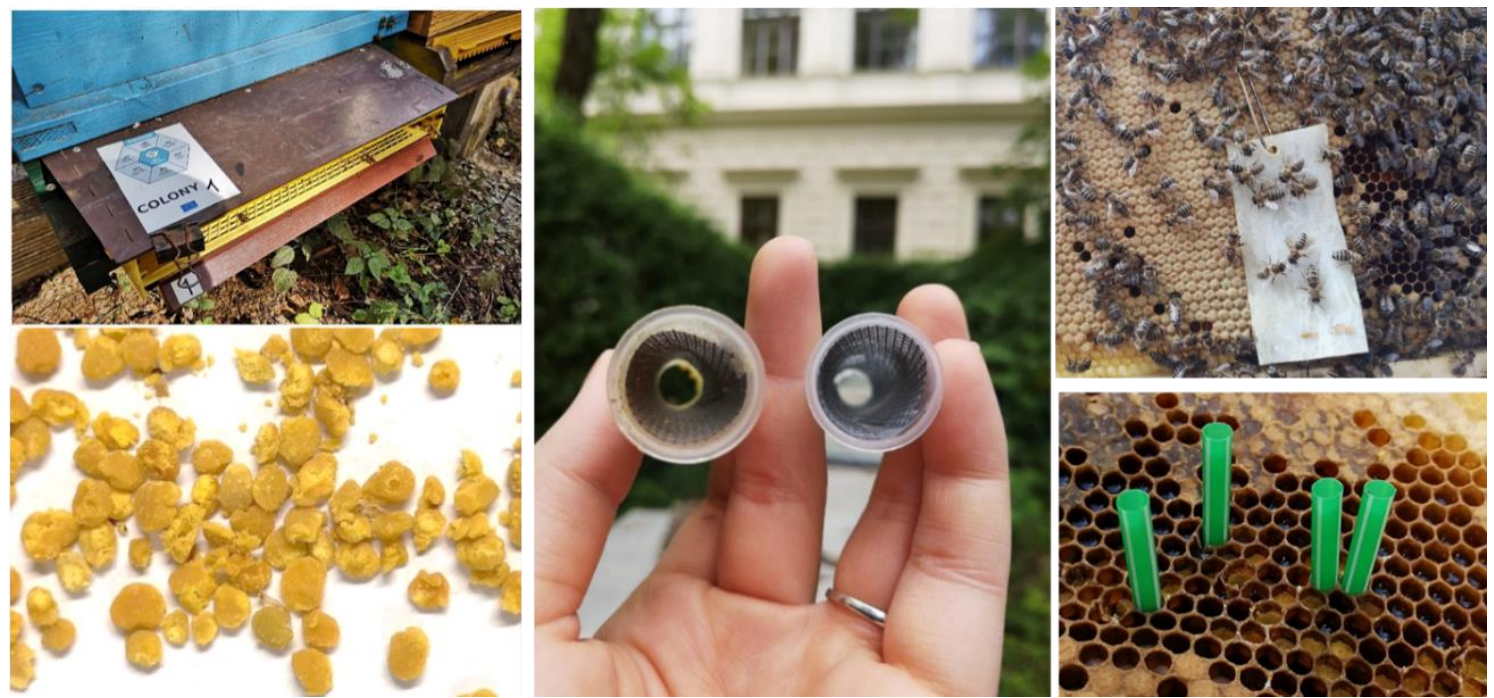

Figure 2. Different sampling techniques applied by citizen science beekeepers in the INSIGNIA study in 2019. Left: Pollen trap (above) and pollen loads (below). Middle: Beehold tubes. Right: APIStrips (above) and sampling beebread with straws.

\subsection{Sample logistics and Murphy's law}

Cooperation with many people inevitably results in many small things not working as planned. These ranged from unreadable labels of sampling bags, because a wrong pen was used (circumvented by printing unique numbers on each label and later by equipping citizen scientists with pencils) to broken equipment due to storms (Figure 3). Unassignable samples were excluded from further analysis and broken equipment was replaced. Beekeepers also demonstrated their craft in finding their own creative solutions to improve the study set-up.
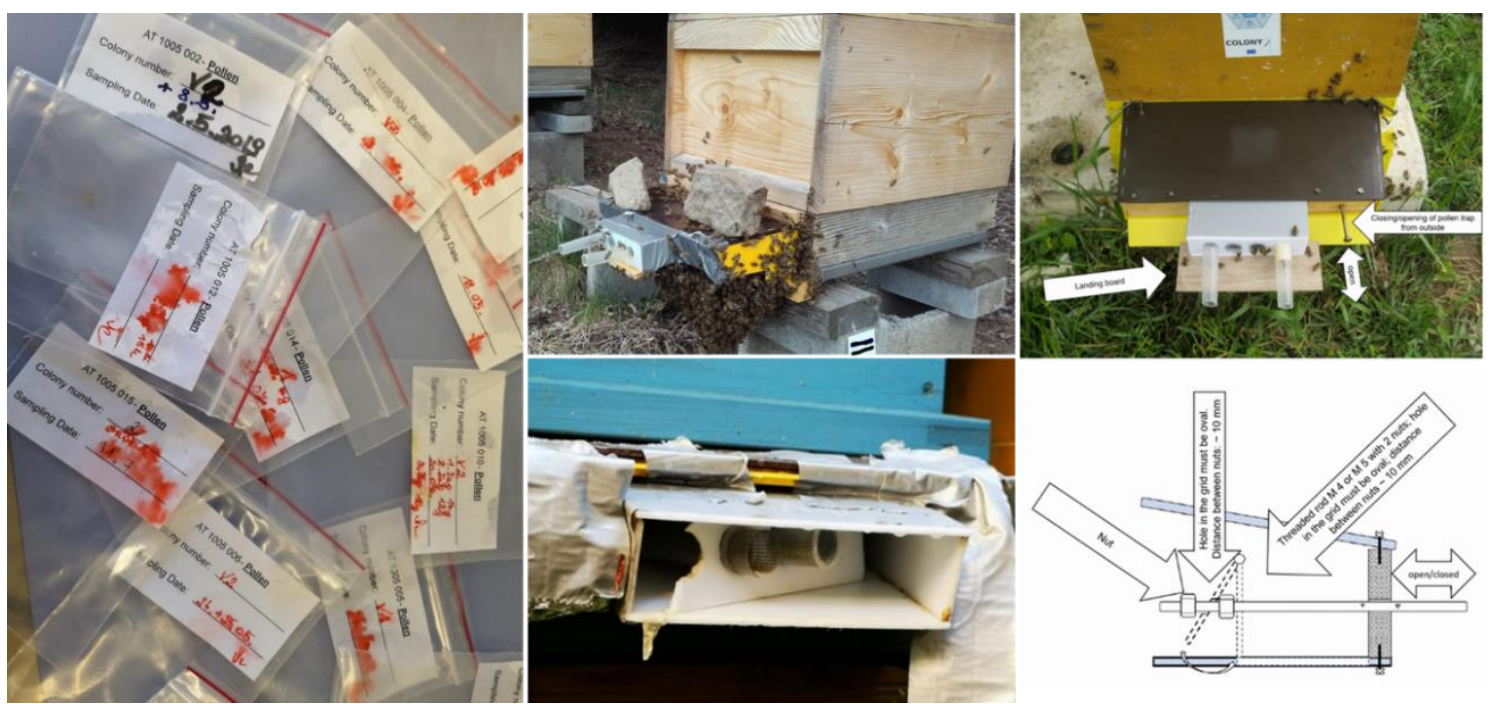

Figure 3. Unreadable labels of sample bags (left), bees not being very cooperative or broken equipment (middle) and citizen scientists' inventiveness: prototype and sketch plan for improving usability of sampling devices (right). 


\subsection{Citizen scientists' profiles and motivation}

In both years, the role and motivation of citizen scientists was studied. In 2019, a trained social scientist interviewed citizen scientists at the beginning of the study and midway or later in the season. The results of this study were presented in a talk at the Austrian citizen science conference 2020 and will be published separately (Bieszczad et al., subm.). The findings of this study helped to streamline the protocol for the second year. In 2020, the larger number of citizen scientists were surveyed online as in the study of Alender [7]. Again, the results of this survey will be published separately (Gratzer and Brodschneider, subm).

\section{Conclusions}

Citizen science studies involving honey bees are not open to every citizen but have the benefit that volunteer beekeepers are equipped and knowledgeable in the field of apiculture. They are neither professional scientists, nor received a salary or compensation in our study, which well qualifies them as citizen scientists. The unique feature of INSIGNIA was that the funding was dedicated to develop methods. This allowed two field seasons, first with a small number of beekeepers trying out four methods. The experience during this first year, and the sociological study helped streamline the study plan for the second field season.

All four sampling methods tested in 2019 have their pros and cons. They differ in user- and bee-friendliness, have distinct temporal resolutions of the information they depict, require different procedures for conservation and storage and differ in price. Price was highest for the two sampling devices, and lowest for the two bee products. Beebread in turn was found to be the least user- and bee-friendly matrix to sample.

The biggest advantage of the public participation is without doubt the investigation of large areas. With the help of beekeepers, sampling sites can be greatly increased without regular travel of researchers to these sites. The physical transport of samples remains one of the biggest challenges, especially if the cool chain needs to be maintained. In the two study seasons, national coordinators either arranged samples to be handed over personally or shipping with cool packs. Given an up-scaling in number of participants, other ways of preserving samples without freezing are currently being studied, so that samples can be shipped via post. For sampling of environmental contaminants, the APIStrips developed in this project have proved reliable [6]. Alternatives for citizen scientists to easily preserve DNA from pollen samples are currently investigated.

\section{References}

[1] C. Porrini, E. Caprio, D. Tesoriero, G. Di Prisco, Using honey bee as bioindicator of chemicals in Campanian agroecosystems (South Italy). Bulletin of Insectology 67 (2014), 137-146.

[2] J.J.M. van der Steen, J. de Kraker, J.T.C. Grotenhuis, Assessment of the potential of honeybees (Apis mellifera L.) in biomonitoring of air pollution by cadmium, lead and vanadium. Journal of Environmental Protection 6 (2015), 96-102. DOI: 10.4236/jep.2015.62011

[3] R. Brodschneider, K. Gratzer, E. Kalcher-Sommersguter, H. Heigl, W. Auer, R. Moosbeckhofer, K. Crailsheim, A citizen science supported study on seasonal diversity and monoflorality of pollen collected by honey bees in Austria. Sci Rep 9 (2019), 16633. DOI:10.1038/s41598-019-53016-5

[4] J.L. Shirk, H.L. Ballard, C.C. Wilderman, T. Phillips, A. Wiggins, R. Jordan, E. McCallie, M. Minarchek, B.V. Lewenstein, M.E. Krasny, R. Bonney, Public participation in scientific research: a 
framework for deliberate design. Ecology and society 17 (2012), 29. DOI: 10.5751/ES-04705170229

[5] J.J.M. van der Steen, Beehold: the colony of the honeybee (Apis mellifera L) as a bio-sampler for pollutants and plant pathogens, Wageningen University (2016). DOI: 10.18174/375348 (2016)

[6] M. Murcia-Morales, J.J.M. van der Steen, F. Vejsnæs, F.J. Díaz-Galiano, J.M. Flores, A.R. Fernández-Alba, APIStrip, a new tool for environmental contaminant sampling through honeybee colonies. Science of The Total Environment (2020), 138948. DOI: 10.1016/j.scitotenv.2020.138948

[7] B. Alender, Understanding volunteer motivations to participate in citizen science projects: a deeper look at water quality monitoring. Journal of Science Communication 15 (2016), A04.

\section{Acknowledgements}

Our thanks are extended to the beekeepers participating in this study. The study received funding from the European Union (Pilot project on environmental monitoring of pesticide use through honey bees No SANTE/2018/E4/SI2.788418-SI2.788452, acronym: INSIGNIA). 